\title{
Resonant excitation of nanowire quantum dots
}

\author{
Lorenzo Leandro', Jacob Hastrup (iD ${ }^{1,2}$, Rodion Reznik ${ }^{3}$, George Cirlin ${ }^{3,4,5}$ and Nika Akopian (iD ${ }^{1 凶}$
}

GaAs quantum dots in nanowires are one of the most promising candidates for scalable quantum photonics. They have excellent optical properties, can be frequency-tuned to atomic transitions, and offer a robust platform for fabrication of multi-qubit devices that promise to unlock the full technological potential of quantum dots. Coherent resonant excitation is necessary for virtually any practical application because it allows, for instance, for on-demand generation of single and entangled photons, photonic clusters states, and electron spin manipulation. However, emission from nanowire structures under this excitation scheme has never been demonstrated. Here we show, for the first time, biexciton-exciton cascaded emission via resonant two-photon excitation and resonance fluorescence from an epitaxially grown GaAs quantum dot in an AIGaAs nanowire. We also report that resonant excitation schemes, combined with above-bandgap excitation, can be used to clean and enhance the emission of nanowire quantum dots.

npj Quantum Information (2020)6:93; https://doi.org/10.1038/s41534-020-00323-9

\section{INTRODUCTION}

$\mathrm{GaAs}^{1,2}$ and $\operatorname{lnGaAs}{ }^{3}$ quantum dots (QDs) are excellent single photons sources in terms of purity and near-unity indistinguishability. GaAs QDs, in particular, have shown the highest degree of photon polarization entanglement ${ }^{4}$. These QDs emit around $780 \mathrm{~nm}$, near the $D_{2}$ lines of ${ }^{87} \mathrm{Rb}$, a stable and reliable reference frequency to which each emitter can be tuned ${ }^{5,6}$ and frequencylocked for quantum network operation ${ }^{7}$. These characteristics make them one of the best candidates for applications in quantum information networks ${ }^{8,9}$.

Resonant excitation of semiconductor QDs ${ }^{10-13}$ allows for ondemand operation, by triggering the emission with precisely timed pumping pulses. Resonance fluorescence (RF) of the exciton has been shown to allow a high-precision linewidth scan of the emission line ${ }^{14}$ and it is necessary to optically initialize ${ }^{15}$, manipulate ${ }^{16}$, and readout ${ }^{17}$ the spin of the exciton. On the other hand, two-photon excitation (TPE) of the biexciton has been shown to be necessary to obtain a clean biexciton emission and a high degree of indistinguishability and entanglement from cascaded emission of GaAs QDs ${ }^{4}$.

In our previous work ${ }^{18}$, we have shown that GaAs QDs can be embedded in AIGaAs nanowires during bottom-up growth in a molecular beam epitaxy reactor, adding new possibilities to the system. For instance, any number of emitters can be precisely positioned within the same nanowire, making the nanowire QDs structure an ideal platform for advanced multi-qubit devices ${ }^{19,20}$ that are not possible today with their bulk counterparts. Also, the precise size and position of each emitter can be controlled during growth, enabling tuning of the emission wavelength and fabrication of complex devices. The combined system of QDs in nanowires also provides additional functional advantages, challenging to obtain with QDs in bulk but achievable with nanowires, as for example high extraction efficiency ${ }^{21,22}$, maximal fiber mode coupling ${ }^{23,24}$, positioning in uniform arrays ${ }^{25,26}$, and scalable integration to photonic circuits ${ }^{27,28}$.

However, no coherent resonant excitation of nanowire QDs has been demonstrated to date. The emission of incoherently non- resonantly excited GaAs QDs has been observed to typically have no clean biexciton emission ${ }^{4}$ and does not allow for the necessary on-demand operation of the emitters. On the other hand, resonant excitation based on cross-polarization schemes requires excellent filtering of the excitation laser. The rough surfaces and the multiple facets of the nanowire, visible for example in Fig. 1a, scramble the wavefront of the scattered laser, producing a nonuniform polarization which reduces the laser rejection and thus makes it more difficult to separate the scattered excitation from the RF signal. Lack of a clean excitation technique strongly limits the usability of nanowires QDs for practical quantum technology applications.

Here we demonstrate resonant TPE of the biexciton and resonant excitation of the exciton in a nanowire QD. We show that the biexciton, which is barely visible under above band-gap excitation, emerges bright with a clean spectrum under resonant TPE and generates a biexciton-exciton cascaded emission to the ground state.

\section{RESULTS AND DISCUSSION}

Optical characterization

In Fig. 1a we show a scanning electron microscope image of a nanowire $\mathrm{QD}$, highlighted in blue. The $\mathrm{GaAs} \mathrm{QD}$, being very small and embedded inside the AIGaAs, is not visible. Also in Fig. 1, we present the optical characterization of a nanowire QD under above bandgap excitation (Fig. 1b, c) and TPE (Fig. 1d, e), at a temperature of $1.5 \mathrm{~K}$. Figure $1 \mathrm{~b}$ shows the energy levels and the relevant transitions under above bandgap excitation, where we draw $\left.|0>,| X^{0}\right\rangle$, and $\left|X X^{0}\right\rangle$, the ground state, neutral exciton and biexciton levels, and $\left|0^{*}\right\rangle$ and $\left|X^{*}\right\rangle$, the state of a QD filled by a single charge and the charged exciton. Under such excitation every level is excited in a non-deterministic way, producing the typical spectrum in Fig. 1c. In most cases, we observe a bright line, the neutral exciton emission $\mathrm{X}^{0}$, and a group of one or more lines at $1-3 \mathrm{~nm}$ longer wavelength than the exciton. In the vast majority of cases, the neutral biexciton emission $\mathrm{XX}^{0}$ is not or

\footnotetext{
'DTU Department of Photonics Engineering, Technical University of Denmark, 2800 Kgs. Lyngby, Denmark. ${ }^{2}$ DTU Department of Physics, Technical University of Denmark, 2800 Kgs. Lyngby, Denmark. ${ }^{3}$ ITMO University, Kronverkskiy pr. 49, St. Petersburg 197101, Russia. ${ }^{4}$ St. Petersburg Academic University, RAS, St. Petersburg 194021, Russia. ${ }^{5}$ St. Petersburg Electrotechnical University “LETI", Prof. Popova 5, St. Petersburg 197376, Russia. ${ }^{\circledR}$ email: nikaak@fotonik.dtu.dk
} 

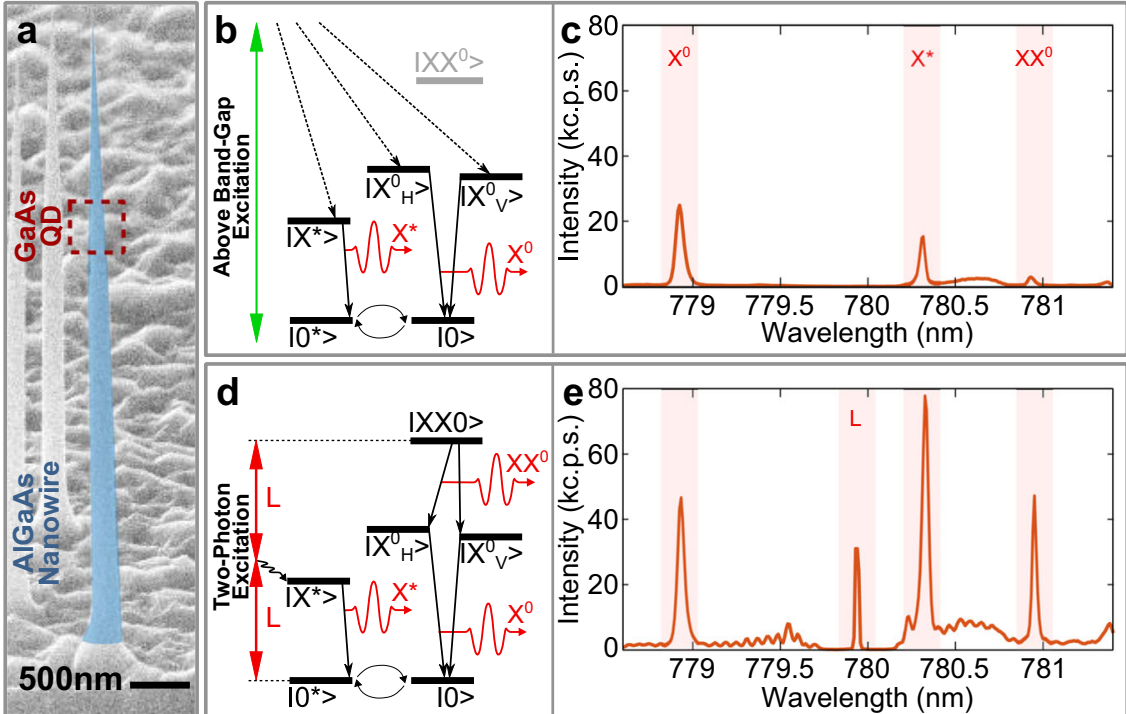

Fig. 1 Two-photon resonant excitation of a nanowire quantum dot. a Scanning electron microscopy image (SEM) showing an AlGaAs nanowire (highlighted blue). The GaAs quantum dot is not visible in SEM images since it is embedded in the nanowire, but its location is marked with a red dashed box. $\mathbf{b}$ and $\mathbf{c}$ Schematics of the energy levels and emission spectrum under continuous-wave above-bandgap excitation. $\mathbf{d}$ and $\mathbf{e}$ Same as in $\mathbf{b}$ and $\mathbf{c}$ but under continuous-wave two-photon excitation of the biexciton. Undulated arrow in $\mathbf{d}$ represents phonon-assisted excitation of the charged exciton.
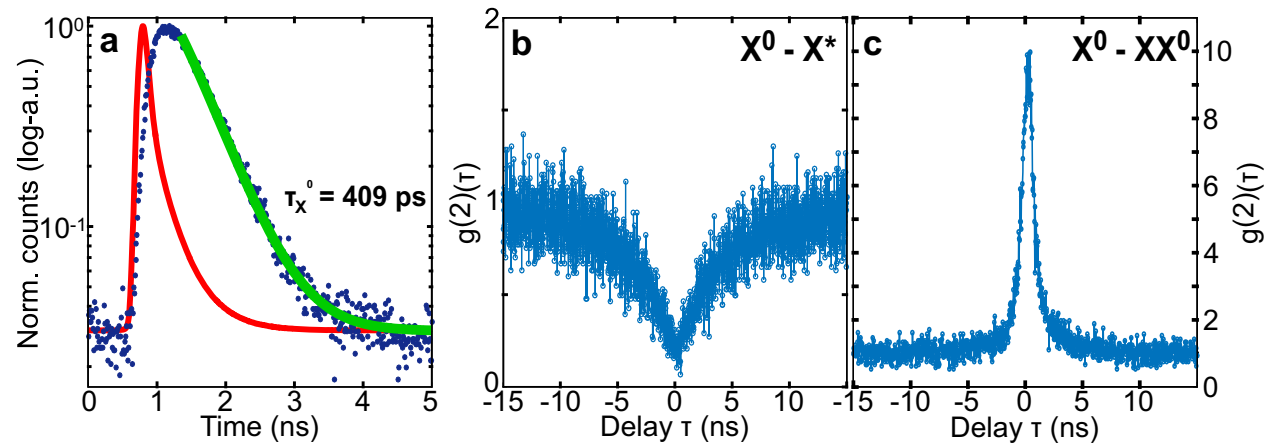

Fig. 2 Lifetime and correlation measurements of a nanowire quantum dot. a Exciton lifetime measurement (blue dots) showing the fast radiative decay time of $409 \pm 11$ ps (fit shown in green), measured under pulsed above-bandgap excitation (excitation laser 730 nm, <3 ps pulse at $76 \mathrm{MHz}$ rate). Also shown, the instrument response function of the setup (red line). $\mathbf{b}$ Cross-correlation measurements of the neutral exciton line with the charged exciton line. c Cross-correlation measurements of the neutral exciton line with the neutral biexciton line showing clear bunching, proving cascaded photon emission. Note that the antibunching cannot be resolved due to the lifetime (409 ps) shorter than the time resolution of our setup (500 ps). Correlation measurements are performed with a narrow $\mathrm{cw} 780 \mathrm{~nm}$ excitation laser. See Supplementary Fig. 1 for the full set of correlation measurements.

barely visible under this type of excitation, while the neutral exciton is always present.

Figure 1e shows the schematic of the energy levels and emission under resonant TPE of the biexciton state $\left|X X^{0}\right\rangle$. In this case, the laser is tuned at half the energy difference between the ground state and the biexciton. This excites $\mid X X^{0}>$ resonantly via TPE producing the spectrum in Fig. 1d, showing identical intensity for the $X^{0}$ and $X X^{0}$ lines. For this experiment, the laser passes through a set of ultra-narrow notch filters each with full width at half maximum (FWHM) of $0.4 \mathrm{~nm}$. The first filtering is done on the excitation path, to clean the laser from its tail to avoid it tampering with the detection of the $\mathrm{X}^{0}$ or $\mathrm{XX}^{0}$ lines. The second filtering is done on the emission path, suppressing the scattered laser by $\sim 7$ orders of magnitude. What remains after filtering is visible in the spectrum as a narrow laser line (L) and a broad modulation coming from the remaining tail of the laser. This type of laser rejection allows performing TPE without the use of crosspolarization setups ${ }^{29,30}$ thus, for instance, allowing for simultaneous measurements of different polarization projections, used in entanglement demonstrations. For this nanowire QD, the laser also excites a charged exciton $X^{*}$. This transition is visible, most probably, due to phonon-assisted excitation ${ }^{31}$. This type of excitation, even if low in efficiency, is a process that requires only one photon and thus results in a bright $X^{*}$ line.

Remarkably, our GaAs nanowire QDs show similar optical characteristics to their bulk counterpart of symmetric GaAs QDs ${ }^{4}$, widely studied for their clear potential for quantum photonic applications.

Time-resolved measurements

In Fig. 2a, we show the measured fast decay of the $X^{0}$ line measured under pulsed above-bandgap excitation. A monoexponential fit, convoluted with the instrument response function of our setup, reveals a radiative recombination lifetime of $409 \pm 11$ ps.

In Fig. 2b, c, we show the cross-correlation measurements of the neutral exciton with charged exciton or neutral biexciton. The antibunching visible in Fig. $2 \mathrm{~b}$ proves the $\mathrm{X}^{0}$ and $\mathrm{X}^{*}$ lines are 


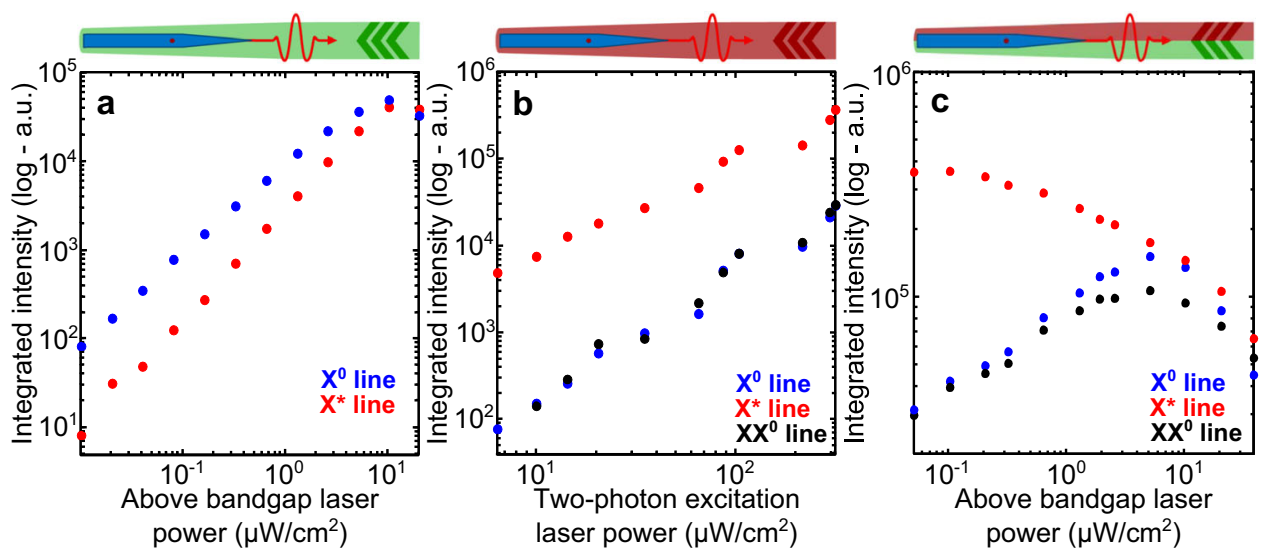

Fig. 3 Excitation power dependencies of above-bandgap and two-photon excitation. Integrated intensities versus power of the excitation laser under a above-bandgap excitation, $\mathbf{b}$ two-photon excitation with resonant laser only, and $\mathbf{c}$ two-photon excitation with fixed laser power, along with the above-bandgap green laser that is varied in power.
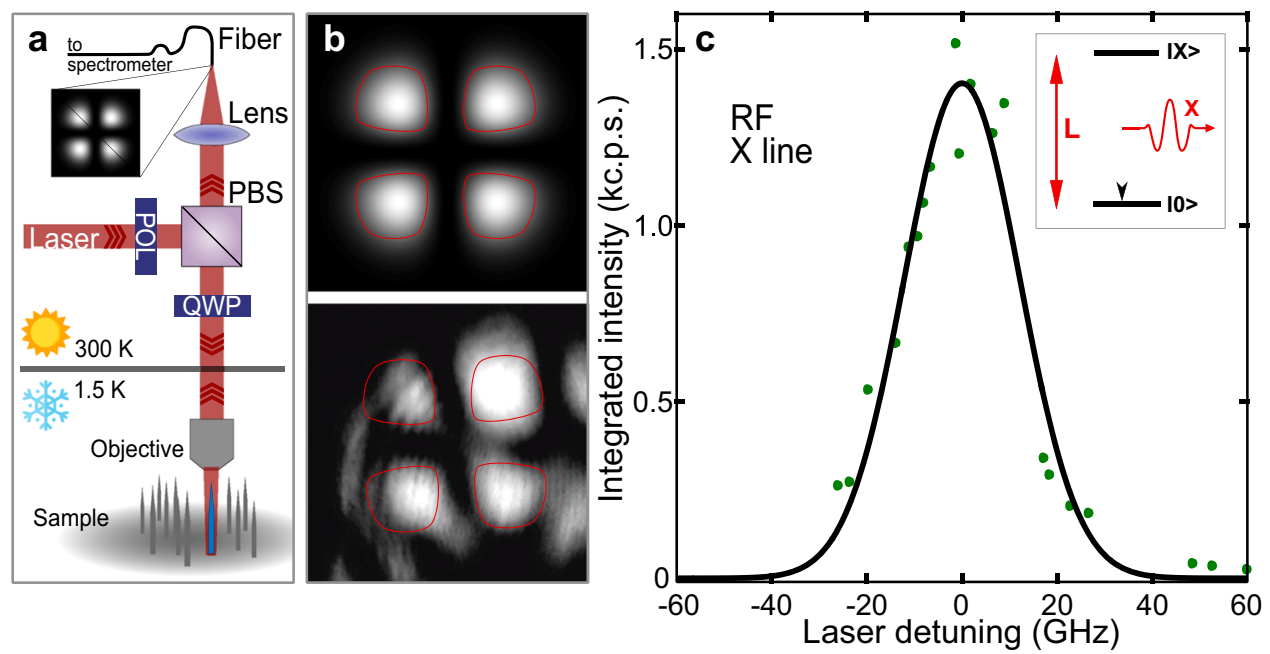

Fig. 4 Resonant fluorescence and linescan of a nanowire quantum dot. a Schematics of the experimental setup, a cryogenic crosspolarization confocal microscope. PBS polarizing beam splitter, POL polarizer, QWP quarter-waveplate. b Simulated (top) and measured (bottom) "flower" patterns of the focused reflected laser. The simulated pattern displayed for illustration (for more details see Supplementary Note 2). The remaining laser intensity is marked red for clarity. The central part of this pattern, containing virtually no laser but most of the resonance fluorescence signal, is focused into a single-mode fiber obtaining a laser rejection of $\sim 6$ orders of magnitude. c Resonant fluorescence (RF) and linescan of a nanowire quantum dot. The narrow laser is scanned through the exciton resonance and the integrated emission is measured (green circles) in the low power regime (see Supplementary Note 3 for background correction). Gaussian fit is shown in black. Inset: Schematics of the involved energy levels.

mutually exclusive and thus coming from the same QD, while its large temporal width, as compared to the exciton lifetime, reveals a few-ns-long charging and discharging mechanisms. Given the fast decay of the exciton, the $X^{0}-X^{0}$ and $X X^{0}-X X^{0}$ antibunching could not be clearly resolved, but the clear bunching in the $\mathrm{X}^{\mathrm{O}}-\mathrm{XX}^{0}$ cross-correlation, shown in Fig. $2 \mathrm{c}$, proves cascaded emission. The full set of correlation measurements can be found in the Supplementary Fig. 1.

\section{Excitation power dependencies}

In Fig. 3 we present the power dependencies of the excitonic lines for both above bandgap and TPE. Figure 3a shows the integrated intensities of $X^{0}$ and $X^{*}$ while varying the power of only the green above bandgap laser. As expected in this case, $X^{0}$ and $X^{*}$ grow with similar rates until they eventually saturate, in the same range of excitation power. Note that the $X X^{0}$ line is not visible under above bandgap excitation, as already observed in epitaxial GaAs $\mathrm{QDs}^{4}$, due to the presence of several multi-excitonic lines that suppress the $X X^{0}$ line. For this reason, TPE is used to directly address the biexciton state.

In Fig. 3b we show the integrated intensity of all three lines versus the power of the resonant two-photon red laser. In this case, we observe an equal rate for $\mathrm{X}^{0}$ and $\mathrm{XX}^{0}$, as expected for cascaded emission at low excitation powers ${ }^{29}$. This behavior is due to the fact that the exciton state is populated only after the decay of the biexciton state and thus we expect one exciton photon for each biexciton photon. The power of the used excitation laser in the current experimental setup is not enough to reach the saturation condition of the excitonic lines.

Lastly, in Fig. 3c, we show the case of fixed-power TPE with additional application of above bandgap pumping. This slight addition is known to enhance the intensity of $X^{0}$ and $X X^{0}$ excited via the TPE process ${ }^{4}$. This mechanism is thought to be due to the stabilization of charges and filling of charge traps in the vicinity of the QD, that could otherwise bring the system in long-lived and non-emitting states ${ }^{32}$. This gating mechanism we observe for both TPE (Fig. 1d) and resonant fluorescence (Fig. 4c). In Fig. 3c, we show 
the effect of such an additional gating laser, which clearly reduces the intensity of $X^{*}$ line in favor of the $X^{0}$ and $X X^{0}$ lines. At high enough powers of the green laser, the $X^{0}$ line is also excited nonresonantly, showing higher intensity than the $X^{0}$ line, just before saturation. We note that this dependence of an additional above-bandgap laser has been observed to change from emitter to emitter. Nonetheless, generally speaking, a very small amount of above bandgap excitation (not enough to directly excite the emitter) results in improved TPE and enhanced emission of $X^{0}$ and $X X^{0}$.

\section{Resonant fluorescence}

In Fig. 4 we show resonant fluorescence measurement of a nanowire QD. A schematic of the measurement configuration used for the experiment is shown in Fig. 4a. This setup, similar to the ones used in previous experimental demonstrations ${ }^{14,33}$ on bulk emitters with flat outer surfaces, uses a cross-polarization scheme that allows to distinguish the scattered excitation laser from the QD emission. The excited QDs will, in most cases, emit a mixture of light horizontal $(\mathrm{H})$ and vertical $(\mathrm{V})$ linearly polarized or right-hand (R) and left-hand (L) circularly polarized, while the laser is strongly linearly polarized in only one direction and can, therefore, be efficiently filtered. After passing through the polarizing beam splitter, the laser focused by the lens shows the typical "flower" pattern (see Fig. 4b and Supplementary Note 2 for details) that comes from the focusing ${ }^{34}$. This flower pattern is a result of the non-perfect polarization filtering of a strongly focused beam but shows still virtually no remaining laser in the center, where most of the QD emission is present. This pattern is aligned with a single mode fiber that acts as a pinhole in our confocal system, resulting in a laser rejection of $\sim 6$ orders of magnitude.

In Fig. $4 c$ we show the integrated intensity of the exciton line of a nanowire QD while scanning the laser through the resonance at an excitation power much lower than the saturation condition. This scan allows us to infer the QD linewidth by a Gaussian fit (assuming inhomogeneous broadening), which shows a FWHM of $118.3 \mu \mathrm{eV}$, far from the lifetime-limited emission linewidth for this dot, due to nearby charge fluctuations ${ }^{35,36}$. During the scan, a minimal amount of above-bandgap laser is also applied, only to gate the emission. This helps because it makes it possible to perform a scan with and without QD emission, allowing to correct for the background coming from the remaining laser (see Supplementary Note 3 for details). This scheme was tested on several nanowire QDs, with results varying mostly on laser rejection and not on the underlying physics.

We note that the exciton linewidth did not vary substantially between the three different excitation techniques (FWHM $110 \mu \mathrm{eV}$ for above bandgap excitation, $104 \mu \mathrm{eV}$ for TPE, and $108 \mu \mathrm{eV}$ for $\mathrm{RF}$ ), while all of them result in a linewidth far from the lifetime limit. This, rather unexpected result, suggests that the limiting factor is intrinsic to the structure and the local environment around the QDs ${ }^{35}$, and not to the excitation process, even if we have shown in our previous work that some QDs show much narrower linewidths, down to $9.4 \mu \mathrm{eV}^{18}$.

\section{Conclusions}

In summary, we have demonstrated resonant excitation of an exciton and biexciton in GaAs nanowire QDs. This was possible thanks to a combination of technological advancements of the nanowire platform (i.e., relatively high brightness and narrow linewidth) and careful optimization of the optical setup. Under two-photon continuous-wave resonant excitation, our nanowire QDs show a clean spectrum and biexciton-exciton cascaded emission. This result is obtained without polarization filtering, allowing for further polarization-resolving measurements, such as entanglement generation, and substantially reducing the setup complexity. A cross-polarization confocal microscope is developed to resonantly drive the exciton, demonstrating resonant fluorescence of a nanowire QD. This allows to directly and individually drive excitonic transitions in nanowire QDs, paving the way to advanced multi-qubit photonic devices where the quantum state of each QD in a nanowire can be individually addressed and manipulated.

\section{METHODS}

Sample growth

We grow our nanowire QDs by Au-catalyzed vapor-liquid-solid (VLS) method in a molecular beam epitaxy reactor on a Si(111) substrate. After a $0.1 \mathrm{~nm} \mathrm{Au}$ deposition at $550^{\circ} \mathrm{C}$, the gold forms droplets of $\sim 20 \mathrm{~nm}$ in diameter. At a temperature of $510^{\circ} \mathrm{C}$, a V/III flux (ratio of 3) is applied to grow $\mathrm{Al}_{0.3} \mathrm{Ga}_{0.7} \mathrm{As}$ nanowires with a diameter set by the gold catalyst. At any given point, the Al flux can be turned off to grow an insertion of GaAs (the QD) for a few seconds. Then, the AIGaAs nanowire growth is resumed. MBE growth of Au-catalyzed AlGaAs nanowires leads to the spontaneous formation of unintentional shell structure around the core. This shell forms via vapor-solid process typically with Al content twice larger than in the core ${ }^{37}$. Thus, AlGaAs overgrowth after the GaAs insertion leads to complete embedding of the QD. Further details and transmission electron microscopy images of the grown nanowire QDs can be found in the literature ${ }^{38}$.

\section{Measurement setup}

All optical measurements are performed at a temperature of $1.5 \mathrm{~K}$ in a closed-cycle He cryostat, developed by Cryovac. The above-bandgap excitation is done by using a continuous wave $532 \mathrm{~nm}$ laser to measure the spectrum or gate the TPE, and with a titanium sapphire (TiSa) picosecond laser, tuned to $730 \mathrm{~nm}$, for the lifetime measurements. The TPE and correlation measurements are done by using a Toptica tunable diode laser (765-805 nm), filtered $\sim 7$ orders of magnitude from the RF signal, with very narrow Optigrate notch filters. All excitation lasers are focused onto the sample with an objective with a numerical aperture of 0.85 , which also collects the emission. The emission signal is then coupled free-space to either one or two $75 \mathrm{~cm}$ spectrometers with a $1800 \mathrm{~g} / \mathrm{mm}$ holographic grating providing a resolution of $\sim 0.01 \mathrm{~nm}$. The spectrometers were equipped with low-noise Peltier-cooled coupled charged devices to measure the photoluminescence spectrum, or with single-photon detectors to measure lifetime or correlations. For lifetime measurements we use a fast micro-photon devices (MPD) detector ( 170 ps FWHM), while for correlation measurements we use two slower Excelitas detectors ( $200 \mathrm{ps}$ FWHM). Taking into consideration the measured lifetime, the setup efficiency and the typical number of photons measured at the spectrometers, we estimated an overall photon extraction efficiency on the order of $1 \%$, for nanowire QDs without any intentional cavity design or tapering optimization. The RF measurement uses the same Toptica laser, but in a different setup: the excitation laser passes through a polarizer, a polarizing beam splitter, and a quarter-waveplate before reaching the sample. The quarter-waveplate is necessary to compensate for any degree of elliptical polarization the beam gathers in the setup because of non-perfect optics. The emission and laser reflection pass through the quarter-waveplate and the polarizing beam splitter and then they are coupled to a single mode fiber and sent to the spectrometer, as shown in Fig. 4a. Following a careful alignment and optimization of the rotating polarizer and waveplate, the scattered laser can be reduced by $\sim 6$ orders of magnitude while keeping most of the photoluminescence signal.

\section{DATA AVAILABILITY}

Data available from the authors upon reasonable request. Correspondence and requests for materials should be addressed to Nika Akopian.

Received: 29 December 2019; Accepted: 2 October 2020; Published online: 04 December 2020

\section{REFERENCES}

1. Schweickert, L. et al. On-demand generation of background-free single photons from a solid-state source. Appl. Phys. Lett. 112, 093106 (2018).

2. Schöll, E. et al. Resonance fluorescence of GaAs quantum dots with near-unity photon indistinguishability. Nano Lett. 19, 2404-2410 (2019). 
3. Senellart, P., Solomon, G. \& White, A. High-performance semiconductor quantumdot single-photon sources. Nat. Nanotechnol. 12, 1026-1039 (2017).

4. Huber, D. et al. Highly indistinguishable and strongly entangled photons from symmetric GaAs quantum dots. Nat. Commun. 8, 15506 (2017)

5. Akopian, N. et al. Tuning single GaAs quantum dots in resonance with a rubidium vapor. Appl. Phys. Lett. 97, 082103 (2010).

6. Akopian, N., Wang, L., Rastelli, A., Schmidt, O. G. \& Zwiller, V. Hybrid semiconductor-atomic interface: slowing down single photons from a quantum dot. Nat. Photonics 5, 230 (2011).

7. Akopian, N. et al. An artificial atom locked to natural atoms. Preprint at https:// arxiv.org/abs/1302.2005 (2013).

8. Duan, L. M., Lukin, M. D., Cirac, J. I. \& Zoller, P. Long-distance quantum communication with atomic ensembles and linear optics. Nature 414, 413-418 (2001).

9. Kimble, H. J. The quantum internet. Nature 453, 1023-1030 (2008).

10. Flagg, E. B. et al. Resonantly driven coherent oscillations in a solid-state quantum emitter. Nat. Phys. 5, 203-207 (2009).

11. Nick Vamivakas, A., Zhao, Y., Lu, C.-Y. \& Atatüre, M. Spin-resolved quantum-dot resonance fluorescence. Nat. Phys. 5, 198 (2009).

12. He, Y.-M. et al. On-demand semiconductor single-photon source with near-unity indistinguishability. Nat. Nanotechnol. 8, 213-217 (2013).

13. Munsch, $M$. et al. Resonant driving of a single photon emitter embedded in a mechanical oscillator. Nat. Commun. 8, 76 (2017).

14. Kuhlmann, A. V. et al. A dark-field microscope for background-free detection of resonance fluorescence from single semiconductor quantum dots operating in a set-and-forget mode. Rev. Sci. Instrum. 84, 073905 (2013).

15. Atatüre, M. et al. Quantum-dot spin-state preparation with near-unity fidelity. Science 312, 551-553 (2006).

16. Press, D., Ladd, T. D., Zhang, B. \& Yamamoto, Y. Complete quantum control of a single quantum dot spin using ultrafast optical pulses. Nature 456, 218-221 (2008).

17. Berezovsky, J. et al. Nondestructive optical measurements of a single electron spin in a quantum dot. Science 314, 1916-1920 (2006).

18. Leandro, L. et al. Nanowire quantum dots tuned to atomic resonances. Nano Lett 18, 7217-7221 (2018)

19. Taherkhani, M., Willatzen, M., Denning, E. V., Protsenko, I. E. \& Gregersen, N. Highfidelity optical quantum gates based on type-II double quantum dots in a nanowire. Phys. Rev. B Condens. Matter 99, 165305 (2019).

20. Hastrup, J., Leandro, L. \& Akopian, N. All-optical charging and charge transport in quantum dots. Sci. Rep. 10, 14911 (2020).

21. Claudon, J. et al. A highly efficient single-photon source based on a quantum dot in a photonic nanowire. Nat. Photonics 1, 215 (2010).

22. Reimer, M. E. et al. Bright single-photon sources in bottom-up tailored nanowires. Nat. Commun. 3, 737 (2012).

23. Bulgarini, G. et al. Nanowire waveguides launching single photons in a Gaussian mode for ideal fiber coupling. Nano Lett. 14, 4102-4106 (2014).

24. Munsch, M. et al. Dielectric GaAs antenna ensuring an efficient broadband coupling between an InAs quantum dot and a Gaussian optical beam. Phys. Rev. Lett. 110, 177402 (2013).

25. Tseng, C.-H., Tambe, M. J., Lim, S. K., Smith, M. J. \& Gradecak, S. Position controlled nanowire growth through $\mathrm{Au}$ nanoparticles synthesized by galvanic reaction. Nanotechnology 21, 165605 (2010).

26. Subannajui, K., Güder, F. \& Zacharias, M. Bringing order to the world of nanowire devices by phase shift lithography. Nano Lett. 11, 3513-3518 (2011).

27. Elshaari, A. W. et al. On-chip single photon filtering and multiplexing in hybrid quantum photonic circuits. Nat. Commun. 8, 379 (2017).

28. Zadeh, I. E. et al. Deterministic integration of single photon sources in silicon based photonic circuits. Nano Lett. 16, 2289-2294 (2016)

29. Ardelt, P.-L. et al. Optical control of nonlinearly dressed states in an individual quantum dot. Phys. Rev. B Condens. Matter 93, 165305 (2016).

30. Hargart, F. et al. Cavity-enhanced simultaneous dressing of quantum dot exciton and biexciton states. Phys. Rev. B Condens. Matter 93, 115308 (2016).

31. Weiler, $S$. et al. Phonon-assisted incoherent excitation of a quantum dot and its emission properties. Phys. Rev. B Condens. Matter 86, 241304 (2012).

32. Nguyen, H. S. et al. Optically gated resonant emission of single quantum dots. Phys. Rev. Lett. 108, 057401 (2012).

33. Ding, $X$. et al. On-demand single photons with high extraction efficiency and near-unity indistinguishability from a resonantly driven quantum dot in a micropillar. Phys. Rev. Lett. 116, 020401 (2016).
34. Novotny, L., Grober, R. D. \& Karrai, K. Reflected image of a strongly focused spot Opt. Lett. 26, 789-791 (2001).

35. Chen, D., Lander, G. R., Krowpman, K. S., Solomon, G. S. \& Flagg, E. B. Characterization of the local charge environment of a single quantum dot via resonance fluorescence. Phys. Rev. B Condens. Matter 93, 115307 (2016).

36. Arnold, C. et al. Cavity-enhanced real-time monitoring of single-charge jumps at the microsecond time scale. Phys. Rev. X 4, 021004 (2014).

37. Dubrovskii, V. G. et al. Origin of spontaneous core-shell AIGaAs nanowires grown by molecular beam epitaxy. Cryst. Growth Des. 16, 7251-7255 (2016).

38. Cirlin, G. E. et al. AlGaAs and AlGaAs/GaAs/AlGaAs nanowires grown by molecular beam epitaxy on silicon substrates. J. Phys. D 50, 484003 (2017).

\section{ACKNOWLEDGEMENTS}

We gratefully acknowledge the support of Villum Fonden (Project no. VKR023444) and Marie \& M.B. Richters Fond (Project no. 17-11014). The nanowire samples were grown under the support of the Russian Science Foundation (Project no. 19-7230004). We acknowledge Cryovac GmbH (www.cryovac.de) for developing a cryogenic system used in some of the experiments reported here. We thank Josh Clement, Jaagup Repän, and Michael Reynolds for their help in the building of the experimental setups and understanding of the emission that led to the results presented in this work. We thank Kai Müller and Jonathan J. Finley for the advice and discussions that helped us designing the resonance fluorescence setup. We also thank Armando Rastelli and Rinaldo Trotta for providing test samples with GaAs QDs in planar epitaxial structures.

\section{AUTHOR CONTRIBUTIONS}

L.L., J.H., and N.A. conceived the original concept and designed the experiment. R.R. and G.C. grew the samples. L.L. and J.H. built the experimental setup, performed the measurements, and analyzed the data. J.H. developed numerical tools and performed the simulation. L.L., J.H. and N.A. discussed the results and wrote the manuscript with input from R.R. and G.C. N.A. coordinated the project.

\section{COMPETING INTERESTS}

The authors declare no competing interests.

\section{ADDITIONAL INFORMATION}

Supplementary information is available for this paper at https://doi.org/10.1038/ s41534-020-00323-9.

Correspondence and requests for materials should be addressed to N.A.

Reprints and permission information is available at http://www.nature.com/ reprints

Publisher's note Springer Nature remains neutral with regard to jurisdictional claims in published maps and institutional affiliations.

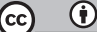

Open Access This article is licensed under a Creative Commons Attribution 4.0 International License, which permits use, sharing, adaptation, distribution and reproduction in any medium or format, as long as you give appropriate credit to the original author(s) and the source, provide a link to the Creative Commons license, and indicate if changes were made. The images or other third party material in this article are included in the article's Creative Commons license, unless indicated otherwise in a credit line to the material. If material is not included in the article's Creative Commons license and your intended use is not permitted by statutory regulation or exceeds the permitted use, you will need to obtain permission directly from the copyright holder. To view a copy of this license, visit http://creativecommons. org/licenses/by/4.0/.

(c) The Author(s) 2020 